\title{
Minimally Invasive Ultrasound-Guided Carpal Tunnel Release: Preliminary Clinical
}

\section{Results}

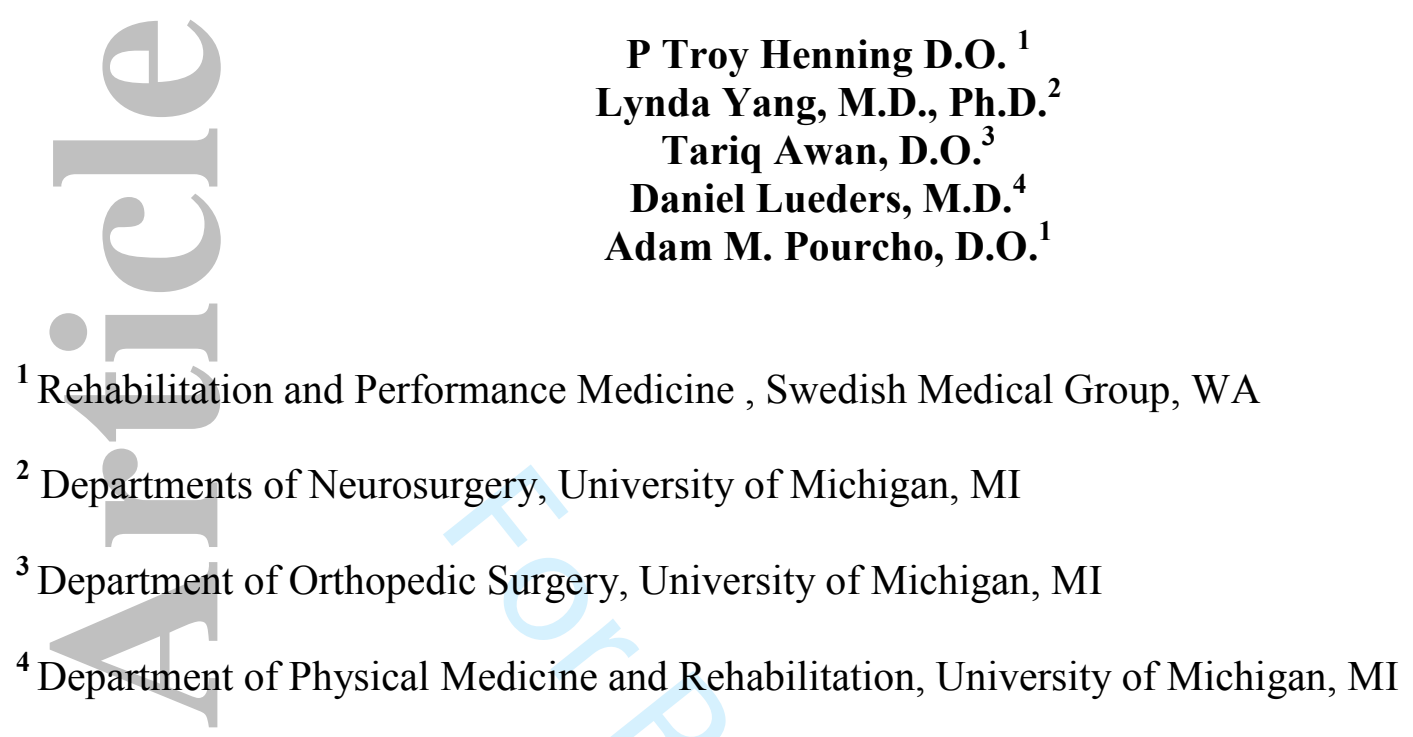

\section{Correspondence:}

P Troy Henning, D.O.

Swedish Rehabilitation and Performance Medicine

Swedish Medical Group

1600 E. Jefferson St. Ste 300

Seattle, WA, 98112

Ph: 425-498-2272

Fax: 425-498-2334

Email: troy.henning@swedish.org

Manuscript type: Case Series

Running title: US Guided Carpal Tunnel Release

Keywords: Ultrasound, Carpal Tunnel Release, Median Nerve,

Abbreviations: CSA - cross sectional area; CTS - carpal tunnel syndrome; CTR - carpal tunnel release; TCL- transverse carpal ligament; TSZ- transverse safe zone; LAX- long axis view; USUltrasound; USCTR - ultrasound-guided carpal tunnel release; USG- ultrasound-guided; SAXShort axis

\section{IRB number: HUM00127628}

Funding: No funding was required for this study

This is the author manuscript accepted for publication and has undergone full peer review but has not been through the copyediting, typesetting, pagination and proofreading process, which may lead to differences between this version and the Version record. Please cite this article as doi:10.1002/ jum.14618. 
USG CARPAL TUNNEL RELEASE 2

\section{Summary}

Ultrasound-guided carpal tunnel release(USCTR) was performed on 14 patients (18 wrists) using dynamic expansion of the transverse safe zone. Our patient population included able bodied and those with impairments. The first 8 cases/12 wrists were performed in an operating room, the remainder in an outpatient setting. No complications occurred, and all patients were able to immediately resume use of their hands without therapy. Improvements in QuickDASH and Boston Carpal Tunnel Questionnaire (BCTQ) at 3 months were comparable to results reported with mini-open and endoscopic release. Our series show USCTR can be safely and effectively performed in an outpatient setting.

\section{INTRODUCTION}

Carpal tunnel syndrome (CTS) is the most common peripheral entrapment neuropathy and has an incidence of $3.5-6.2 \%$, leading to 450,000 surgical releases annually at a total cost of over 2 billion dollars. ${ }^{1-3}$ Greater than $90 \%$ of patients report clinical improvement following release. ${ }^{4-6}$ Although initially performed via a large $(3-5 \mathrm{~cm})$, palmar incision, carpal tunnel release (CTR) techniques have continually evolved to reduce surgical trauma, with the goal of improving cosmesis, reducing post-operative pain, and promoting faster recovery. ${ }^{7}$ Currently available CTR techniques include mini-open CTR (mOCTR) via a single, 1-3 cm palmar incision, endoscopic CTR (ECTR) via one (wrist) or two (wrist and palm) 1-2 cm incisions, and ultrasound-guided CTR (USCTR) via a single $<1 \mathrm{~cm}$ wrist or palmar incision. ${ }^{8-10}$

Regardless of technique, the primary goal of CTR is to transect the transverse carpal ligament (TCL) while avoiding injury to nearby neurovascular structures. ${ }^{11}$ Although ECTR 
USG CARPAL TUNNEL RELEASE 3

may promote a faster recovery compared to mOCTR, concerns have been raised regarding the potential for increased complications due to limited visualization of surrounding structures during TCL transection. ${ }^{10-12}$ USCTR techniques combine a single small incision with direct US visualization of at-risk structures, such as the median nerve and its thenar motor branch/recurrent motor branch, ulnar vessels, and superficial palmar arterial arch. ${ }^{13-20}$ To date, over 620 cases of USCTR have been reported in the peer-reviewed literature with a $>98 \%$ clinical success rate and no documented neurovascular injuries. ${ }^{7,21-26}$ Furthermore, a recently published, single surgeon, prospective randomized clinical trial comparing mOCTR to USCTR reported that patients treated with USCTR experienced significantly faster functional recovery, pain reduction, and pain medication discontinuation. ${ }^{7}$

To transect the TCL, ECTR techniques and most USCTR techniques place the surgical device within the transverse safe zone (TSZ), a region bordered radially by the median nerve and ulnarly by the hook of the hamate or ulnar vessels, whichever lies more radially., ${ }^{7,21,23,24,27,28}$ The TSZ width can range from 4-8 $\mathrm{mm}$ but exhibits significant inter-individual variability and in some individuals can be $<3 \mathrm{~mm}$, or even $0 \mathrm{~mm} .{ }^{21}$ Transection of the TCL through a small skin incision, while at the same time establishing an acceptable TSZ, is a technical challenge with both ECTR and USCTR. During ECTR, both the minimal incision size and the maximally available TSZ are determined by the size of the introducer sheath and endoscopic cannula. In contrast, previously described USCTR techniques require only a very small $(<1 \mathrm{~cm})$ skin incision, but can challenge the operator to establish and maintain an acceptable TSZ. 7,21,23,24,27 A single use, disposable device (SX-One MicroKnife, Sonex Health LLC, Rochester, $\mathrm{MN}$ ) has recently become commercially available. The device allows the user to establish and maintain an acceptable TSZ, via the use of expandable protective balloons. The device is inserted 
USG CARPAL TUNNEL RELEASE 4

through a single $<5 \mathrm{~mm}$ distal forearm skin incision and into the carpal tunnel TSZ, under direct USG. The protective balloons allow for a consistent TSZ of approximately $8 \mathrm{~mm}$ while a retractable microknife cuts the TCL 4-6 mm radial to the hook of the hamate. A recently performed cadaveric investigation using the same device to perform USCTR in 34 unembalmed, cadaveric specimens documented a $100 \%$ rate of TCL release and no neurovascular injuries as assessed by independent observers. $^{29}$

The primary purpose of this manuscript is to document our initial intermediate-term (3 month) clinical results performing USCTR using enhanced transverse safe zone control in a consecutive group of 14 patients (18 wrists). We hypothesized that USCTR using enhanced transverse safe zone control can be performed safely and effectively in an office or procedureroom setting and will allow patients to rapidly recover, including those patients dependent on their upper limbs for ambulation.

\section{METHODS}

A total of 16 patients (22 wrists), ages 62-90 (mean 64 years), with carpal tunnel syndrome were recruited for USCTR from the Physical Medicine and Rehabilitation clinic at the lead author's institution. Two patients/4 wrists were lost to follow-up 4 weeks post-release and are therefore not included in this report. As of their last follow-up point 4 weeks after release these two patients had reported no complications and were recovering uneventfully. Therefore, 14 patients/18 wrists were followed for 12 weeks after release. Patients included able-bodied individuals and those with functional impairments, specifically one patient with post-polio syndrome who ambulated with forearm crutches, one with multiple sclerosis who did not use assistive devices, and two with paraplegia who ambulated with a manual wheelchair. Selection 
USG CARPAL TUNNEL RELEASE 5

criteria included history and examination consistent with CTS, electrodiagnostic testing confirming median neuropathy at the wrist and persistent, functionally limiting symptoms for $>3$ months despite non-operative treatment including activity modification and/or splinting or corticosteroid injections. All procedures were performed by a single fellowship-trained physiatrist $(\mathrm{TH})$ with more than 8 years of clinical experience in diagnostic and interventional musculoskeletal ultrasound (US). The initial 8 cases (12 wrists, 4 bilateral and 4 unilateral) were performed in the Operating Room (OR) in conjunction with a fellowship-trained Neurosurgeon (LY) (not scrubbed in but present for supervision). While in the OR, all standard protocols were followed with respect to sterile preparation and anesthesia, including the use of conscious sedation with appropriate monitoring. The subsequent 8 cases ( 10 wrists, 2 bilateral and 6 unilateral) were performed in an outpatient setting solely by the primary author following standard protocols for outpatient local anesthesia (i.e. no conscious sedation). This manuscript presents the prospective 3-month results of a 12-month study investigating the safety and clinical outcomes of the procedure. All patients were followed using validated outcome measures (the Boston Carpal Tunnel Questionnaire (BCTQ) and Quick form of the Disabilities of the Arm, Shoulder and Hand index (QDASH). The pre-procedure results were collected on the day of the release. One and three-month post- procedure data were collected via mailed questionnaires or follow up phone calls by office staff. One set of scores was collected at each interval regardless if the patient underwent unilateral or bilateral releases.

The BCTQ and QDASH are measures of pain and function commonly used in the evaluation of interventions related to CTS. ${ }^{30}$ The BCTQ has a calculated symptom score (BCTQ-SS) and a functional status score (BCTQ-F) which range 1-5. The QDASH score ranges from 0 -100. Higher scores for both instruments indicate worse symptoms and poorer functional 
USG CARPAL TUNNEL RELEASE 6

status. In addition, two non-validated global outcome measures were utilized at 3-month followup, assessing if symptoms improved (yes/no) and how satisfied patients were with the results of the procedure $(5$ point ordinal scale: $1=$ very dissatisfied, $2=$ dissatisfied, $3=$ neither satisfied nor dissatisfied, $4=$ satisfied, 5 = very satisfied). This study was performed in accordance with the Declaration of Helsinki. This human study was approved by Institutional Review Board of the University of Michigan Medical School (IRBMED) - approval: HUM00127628. All adult participants provided written informed consent to participate in this study.

Prior to USCTR all wrists were evaluated using high frequency transducers on US equipment available in the Department of Physical Medicine and Rehabilitation (15-6 MHz linear array transducer on a Sonosite X-Porte, Fujifilm Sonosite, Bothell, WA, or a 18-5 MHz linear array Philips Affinity, Philips Corporation, Bothell WA). This evaluation utilized standardized protocols including identification of the thenar motor/recurrent motor and palmar cutaneous branches of the median nerve, and any communicating branches between the median and ulnar common digital nerves (so called Berrettini branches) 17,31. The purpose of this evaluation was not only to assess the median nerve but also to identify contraindications to proceeding with USCTR, which included: (a) Inability to adequately visualize at-risk structures including the thenar motor branch/recurrent motor branch of the median nerve, palmar cutaneous branch of the median nerve, ulnar vessels, superficial palmar arterial arch, and median and ulnar palmar digital nerves, (b) Distorted or variant anatomy that would preclude establishment of a safe TSZ, or (c) Presence of mass lesion or other process that requires treatment beyond transection of the TCL. No patients were excluded based on US findings.

Procedure Description 
USG CARPAL TUNNEL RELEASE 7

All USCTR procedures were performed by the primary author using the MicroKnife device in accordance to the manufacturer's instructions, the high frequency linear array transducers previously described, and in accordance to manufacturer's instructions. All patients received an USG local anesthetic injection of approximately $8 \mathrm{~mL}$ of $1 \%$ lidocaine without epinephrine via a 25 -gauge $50 \mathrm{~mm}$ needle. Care was taken to generously infiltrate the planned incision site at the proximal wrist crease (Figure 1) and the palmar fascia superficial to the TCL. This needle was then withdrawn, and a second 25-gauge $50 \mathrm{~mm}$ needle was used to hydrodissect the median nerve away from the flexor tendons and TCL using $10 \mathrm{ml}$ of sterile $0.9 \%$ normal saline under direct ultrasound guidance (USG). This allowed for better delineation of the boundaries of the TCL. Then under ultrasound guidance, a \#15 blade scalpel was used to make vertical stab incision through the pre-anesthetized skin wheal down to and through the antebrachial fascia. Following this, USG was used to accurately place the device into the carpal tunnel, within the TSZ and position the tip just distal to the distal aspect of the TCL. The tip of the device was positioned superficial to the mid-palmar fat pad and proximal and superficial to the superficial palmar arterial arch, approximately $1 \mathrm{~cm}$ distal to the hook of the hamate. Evaluation of the device's position in and out of plane relative to the transducer performed to ensure proper positioning of the device immediately deep to the TCL and radial to the pisiform and hamate. ( Figure 2A-C) The handle on the device was then depressed and locked, filling the balloons to expand and maintain the TSZ. (Figure 2D and supplementary video 1) The device was manipulated slightly ulnarly or radially to optimally position the cutting knife (once activated) away from the path of the median nerve and the ulnar neurovascular structures. Once appropriate positioning in the TSZ was again sonographically confirmed, the knife was deployed and translated in a distal-to-proximal direction under direct USG to transect the TCL (Figure 3A- 
USG CARPAL TUNNEL RELEASE 8

B and supplementary video 2). The knife was then advanced back to its distal location, the regional anatomy re-checked using ultrasound, and a second pass was completed with the knife to ensure complete TCL release. Following complete release. the knife was recessed into its protected position and the protective balloons were deflated. The position of the device was evaluated via US to confirm a now superficial location relative to the TCL and osseous boundaries of the carpal tunnel. The TCL was then probed with the device through the area of transection to ensure no remaining intact fibers persisted (Figure 4 and Supplementary video 3). Probing confirmed complete TCL transection in all cases, and no additional passes were required. The device was then removed from the incision, excess fluid and blood was expressed through the wound followed by adhesive bandages (Steri-Strips) or 4-0 nylon suture closure. (Figure 5) A sterile gauze pad and occlusive dressing were applied. The average total time of the procedure and balloon inflation times were 7 minutes and 2 minutes respectively and the incision length was $<5 \mathrm{~mm}$.

Post-procedure neurologic and physical exam were performed to ensure no neurologic or tendon injuries had occurred. All patients were discharged home under their own power at the same level of pre-procedure function. No activity limitations were imposed and none of the patients required a therapy referral post release. One patient that used bilateral forearm crutches and two that used manual wheelchairs were able to immediately resume the use of these ambulatory aides after release.

\section{Results}

All 16 patients/22 wrists were evaluated in person by the operator one-week post-procedure. There were no complications and all wounds had healed by the first post-operative visit. All 
USG CARPAL TUNNEL RELEASE 9

patients had reported full use of their hands since the day of the procedure. As previously stated, 2 patients/4 wrists were lost to follow-up thereafter and were not included in the formal data analysis. Patients were then mailed the BCTQ and QDASH forms and the two patient oriented outcome measures to collect the 3-month interval data. Those that did not respond by mail were contacted by office staff and completed the surveys over the phone. At one and three months, the average BCTQ-SS score improved from 2.96 pre-release to 1.75 and 1.54 respectively (difference 1.42), and the average BCTQ-F status improved from 2.64 pre-release to 1.75 at one and 3 months (difference 0.89). (Table 1) The average QDASH scores improved from 45 to 19 (one month) and 18 (3 month) (difference 27). (Table 2) All patients reported improvements in symptoms and the average global satisfaction rating was 4 . Importantly, all the patients with impairments retained their pre-procedure level of independence throughout this initial follow-up period.

While not formally collected, we did solicit feedback from patients regarding their experience with USCTR. In general, patients described minimal post-operative pain. Most patients reported adequate pain relief with ice, limb elevation and over the counter analgesic/anti-inflammatory medications. Additionally, patients who received USCTR in the office setting using only local anesthesia generally reported little discomfort throughout the procedure. In this setting many patients also volunteered their satisfaction with the convenience of the procedure with regard to having the procedure done in the outpatient setting, the ease of scheduling, the lack of need to modify medications or dietary intake on the day of the procedure, and the ability to immediately resume use of affected limb.

\section{Discussion}


USG CARPAL TUNNEL RELEASE 10

The most important finding of the current report is that USCTR using enhanced transverse safe zone control can be safely implemented in both the OR and outpatient settings with excellent intermediate-term results in a diverse patient population. In our initial group of 14 patients/18 wrists there were no complications and clinical improvement was similar to that previously reported for mOCTR, ECTR, and USCTR. ${ }^{5,7}$ More specifically, the 3-month improvements in BCTQ-SS and BCTQ-F scores in our cohort were 1.42 and 0.89 , respectively, which exceed the minimally clinically important differences (MCIDs) of 1.14 and 0.74 previously reported by Kim et al. following limited open carpal tunnel release. ${ }^{32}$ Similarly, it has also been reported by Clement et al. that patient satisfaction 3-months following OCTR highly correlated with a post-release QDASH of $\leq 34$ or a post-CTR reduction of $>20$ points. ${ }^{30}$ In the cohort presented in this manuscript, the average 3-month QDASH was 18 and average reduction was 27 , reflecting high patient satisfaction.

Currently, most CTRs are performed using the mOCTR approach with predictably good results and a low complication rate. ${ }^{11,12}$ However, the palmar incisions used for mOCTR may slow recovery and necessitate significant alterations in hand and upper limb function during recovery. 7,11,12 This can lead to increased time away from work or a short stay at a skilled nursing facility for those dependent on use of their upper limbs for transferring and ambulation. Indeed, 3 patients presented in this study were crutch or wheelchair ambulators and were able to transfer and ambulate immediately post-procedure. Smaller incision sizes and reduced procedural trauma appear to promote faster recovery. Studies have demonstrated that patients treated with ECTR or USCTR recover faster and may have less post-procedural pain compared to those treated with mOCTR, presumably in part due to reduced surgical trauma. ${ }^{12}$ These observations have resulted in the historical trend of using smaller palmar incisions for OCTR (i.e. mOCTR). However, the 
USG CARPAL TUNNEL RELEASE 11

smaller palmar incisions used during mOCTR may make the procedure more technically challenging and still require temporary avoidance of palmar pressure. Although ECTR appears to promote a faster recovery and may allow immediate palmar "weightbearing" (in the single incision technique), ECTR is more difficult to perform outside of the operating room setting. ECTR is also more expensive than mOCTR and has been associated with a higher risk of neuropraxia, presumably due to the relatively large size of the cannula and lack of complete visualization of surrounding structures. ${ }^{11,33}$ USCTR provides the advantages of ECTR while allowing the operator to perform the procedure in the office based setting using real-time visualization of all relevant structures. Innovations in image-guided procedures (trigger digit release, tenotomies, fasciotomies and neurolysis) have advanced in-parallel with improved US technology, accessibility, and skilled operators. ${ }^{34,35}$ USCTR has been evolving in the past two decades but has primarily relied on instruments borrowed or repurposed from other procedures, for example, the hook knife. ${ }^{23,36,37}$ USCTR using enhanced transverse safe zone control is the latest advancement in this field and is distinguished by providing the user the ability to access the carpal tunnel via a small forearm incision, control the TSZ via inflatable balloons, and efficiently cut the TCL in a variety of practice settings.

Study strengths include the prospective collection of data using validated patient reported outcome measures, a high follow-up rate $(>87 \%)$ and inclusion of patients with physical impairments dependent on upper limb function for mobility. Nonetheless, we acknowledge several limitations of the current report. First, neither the patient, nor the assessors were blinded to the intervention and there was no comparative or control group. However, the primary purpose of this investigation was to report our initial experience using USCTR with enhanced transverse safe zone control in a clinically relevant setting, including a diverse patient population 
USG CARPAL TUNNEL RELEASE 12

and the use of local anesthesia only. Future studies may include direct comparisons with alternative CTR techniques. Second, we are reporting our intermediate results at 3-month postprocedure. While this follow-up period may be short, it is sufficient to document the safety of USCTR with enhanced transverse safe zone control as used to treat our patients. Furthermore, since $100 \%$ of patients returned to normal activities within the follow-up period, this duration of time was sufficient to document the efficacy of the treatment, as reflected in previous studies. ${ }^{7,22}$ Nonetheless, we will continue to follow our patients for 12-months as previously stated. Third, our sample size is relatively small and include patients treated by a single operator. Future studies should include larger sample sizes and possibly combine experiences from multiple users.

Our initial experience with USCTR using enhanced transverse safe zone control demonstrates that this procedure can be safely and effectively performed in an outpatient setting by physicians who have expertise in advanced ultrasound guided procedures. Specific advantages of USCTR as implemented in our practice include but are not limited to: (1) increased patient convenience of office/procedure room setting using only local anesthesia, freeing up ORs for more complex cases (2) use of small, forearm incision with the ability to immediately use the upper limbs, (3) improved user and patient confidence given the ability to directly visualize all carpal tunnel structures using US and control the TSZ via the use of the inflatable balloons, and (4) reduced need for support personnel following transition to the clinic, accompanied by potential cost savings. The convenience of the office/procedure room and ability to immediately weight bear on the upper limbs are particularly advantageous to patients with functional limitations, as was the case for 3 of our patients. Further assessment of USCTR 
using enhanced transverse safe zone control is justified and should include the assessment of societal implications from both the psychosocial and cost of care perspectives.

\section{Bibliography}

1. Nordstrom DL, DeStefano F, Vierkant RA, Layde PM. Incidence of Diagnosed Carpal Tunnel Syndrome in a General Population. Epidemiology. 1998;9(3):342-345.

2. De Krom MCTFM, Knipschild PG, Kester ADM, Thijs CT, Boekkooi PF, Spaans F. Carpal tunnel syndrome: Prevalence in the general population. Journal of Clinical Epidemiology. 1992;45(4):373.

3. Mondelli M, Giannini F, Giacchi M. Carpal tunnel syndrome incidence in a general population. Neurology. 2002;58(2):289-294.

4. Fajardo M, Kim SH, Szabo RM. Incidence of carpal tunnel release: trends and implications within the United States ambulatory care setting. 2012;37(8):15991605.

5. Huisstede BM, Randsdorp MS, Coert JH, Glerum S, van Middelkoop M, Koes BW. Carpal tunnel syndrome. Part II: effectiveness of surgical treatments--a systematic review. Archives of physical medicine and rehabilitation. 2010;91(7):1005-1024.

6. Palmer DH, Hanrahan LP. Social and economic costs of carpal tunnel surgery. Instructional course lectures. 1995;44:167-172.

7. Rojo-Manaute JM, Capa-Grasa A, Chana-Rodriguez F, et al. Ultra-minimally invasive ultrasound guided carpal tunnel release: a randomized controlled trial. Journal of Ultrasound in Medicine. 2016;35(6):1149-1157.

8. Kim PT, Lee HJ, Kim TG, Jeon IH. Current approaches for carpal tunnel syndrome. 2014;6(3):253-257.

9. Papatheodorou LK, Sotereanos DG. Treatment recommendations for carpal tunnel syndrome and peripheral nerve repair. 2015;64:273-280.

10. Hansen TB, Majeed HG. Endoscopic carpal tunnel release. Hand clinics. 2013;30(1):47-53.

11. Benson LS, Bare AA, Nagle DJ, Harder VS, Williams CS, Visotsky JL. Complications of endoscopic and open carpal tunnel release. Arthroscopy. 2006;22(9):919-924, 924 e911-912.

12. Sayegh ET, Strauch RJ. Open versus Endoscopic Carpal Tunnel Release: A Metaanalysis of Randomized Controlled Trials. Clinical Orthopaedics and Related Research®. 2015;473(3):1120-1132.

13. Rowe NM, Michaels J, Soltanian H, Dobryansky M, Peimer CA, Gurtner GC. Sonographically Guided Percutaneous Carpal Tunnel Release. Annals of Plastic Surgery. 2005;55(1):52.

14. Aydin K, Cokluk C, Piskin A, Kocabicak E. Ultrasonographically checking the sectioning of the transverse carpal ligament during carpal tunnel surgery with limited uni skin incisions. 2007;17(3):219-223. 
15. Abicalaf CA, de Barros N, Sernik RA, et al. Ultrasound evaluation of patients with carpal tunnel syndrome before and after endoscopic release of the transverse carpal ligament. Clinical radiology. 2007;62(9):891-894.

16. Lecoq B, Hanouz N, Vielpeau C, Marcelli C. Ultrasound-guided percutaneous surgery for carpal tunnel syndrome: a cadaver study. Joint Bone Spine. 2011;78(5):516-518.

17. Smith J, Barnes DE, Barnes KJ, et al. Sonographic Visualization of Thenar Motor Branch of the Median Nerve: A Cadaveric Validation Study. PM \& $R$ : the journal of injury, function, and rehabilitation. 2016;9(2):159-169.

18. Dineen JM, Greenberg SA. What role for ultrasound in diagnosing carpal tunnel syndrome? Pract Neurol. 2014;14(5):286-287.

19. Chern TC, Jou IM, Chen WC, Wu KC, Shao CJ, Shen PC. An ultrasonographic and anatomical study of carpal tunnel, with special emphasis on the safe zones in percutaneous release. 2009;34(1):66-71.

20. de la Fuente J, Miguel-Perez MI, Balius R, Guerrero V, Michaud J, Bong D. Minimally invasive ultrasound-guided carpal tunnel release: a cadaver study. 2013;41(2):101107.

21. Nakamichi K, Tachibana S, Yamamoto S, Ida M. Percutaneous carpal tunnel release compared with mini-open release using ultrasonographic guidance for both techniques. The Journal of Hand Surgery. 2010;35(3):437-445.

22. Lecoq B, Hanouz N, Morello R, et al. Ultrasound-assisted surgical release of carpal tunnel syndrome: results of a pilot, open-label uncontrolled trial conducted outside of the operating room. . Joint Bone Spine. 2015;82(6):442-445.

23. Capa-Grasa A, Rojo-Manaute JM, Rodriguez FC, Martin JV. Ultra minimally invasive sonographically guided carpal tunnel release: an external pilot study. Orthop Traumatol Surg Res. 2014;100(3):287-292.

24. Guo D, Tang Y, Ji Y, Sun T, Guo J, Guo D. A non-scalpel technique for minimally invasive surgery: percutaneously looped thread transection of the transverse carpal ligament. Hand (New York, NY). 2015;10(1):40-48.

25. Buncke G, McCormack B, Bodor M. Ultrasound-guided carpal tunnel release using the manos CTR system. Microsurgery. 2013;33(5):362-366.

26. Chern TC, Wu KC, Huang LW, et al. A cadaveric and preliminary clinical study of ultrasonographically assisted percutaneous carpal tunnel release. Ultrasound in Medicine \& Biology. 2014;40(8):1819-1826.

27. Tachibana K, Nakamichi, S. Ultrasonographically assisted carpal tunnel release. The Journal of Hand Surgery. 1997;22(5):853-862.

28. Tachibana K, Nakamichi S. Distance between the median nerve and ulnar neurovascular bundle: clinical significance with ultrasonographically assisted carpal tunnel release. The Journal of Hand Surgery. 1998;23A(5):870-874.

29. Presley J, Smith J, Barnes DE, Lachman N, Kakar S, Sellon JL. Ultrasound Guided Carpal Tunnel Release (USCTR) Using Dynamic Safe Zone Expansion: A Cadaveric Validation Study. Clinical Journal of Sport Medicine. 2017;27(2):221.

30. Clement ND, Duckworth AD, Jenkins PJ, McEachan JE. Interpretation of the QuickDASH score after open carpal tunnel decompression: threshold values associated with patient satisfaction. Journal of Hand Surgery (European Volume). 2016;41(6):624-631. 
31. Tagliafico A, Pugliese F, Bianchi S, Bodner G, Padua L, Rubino M. High-resolution sonography of the palmar cutaneous branch of the median nerve. American Journal of Roentgenology. 2008;191(1):107-114.

32. Kim JK, Jeon SH. Minimal clinically important differences in the Carpal Tunnel Questionnaire after carpal tunnel release. Journal of Hand Surgery (European Volume). 2012;38(1):75-79.

33. Zhang S, Vora M, Harris AHS, Baker L, Curtin C, Kamal RN. Cost-Minimization Analysis of Open and Endoscopic Carpal Tunnel Release. The Journal of bone and joint surgery American volume. 2016;98(23):1970-1977.

34. Lueders DR, Sellon JL, Smith J, Finnoff JT. Ultrasound-Guided Fasciotomy for Chronic Exertional Compartment Syndrome: A Cadaveric Investigation. $P M R$. 2017;9(7):683-690.

35. Nikolaou VS, Malahias MA, Kaseta MK, Sourlas I, Babis GC. Comparative clinical study of ultrasound-guided A1 pulley release vs open surgical intervention in the treatment of trigger finger. World J Orthop. 2017;8(2):163-169.

36. Nakamichi K-i, Tachibana S. Ultrasonographically assisted carpal tunnel release. The Journal of hand surgery. 1997;22(5):853-862.

37. Petrover D, Silvera J, De Baere T, Vigan M, Hakimé A. Percutaneous UltrasoundGuided Carpal Tunnel Release: Study Upon Clinical Efficacy and Safety. Cardiovascular and interventional radiology. 2016;40(4):568-575.

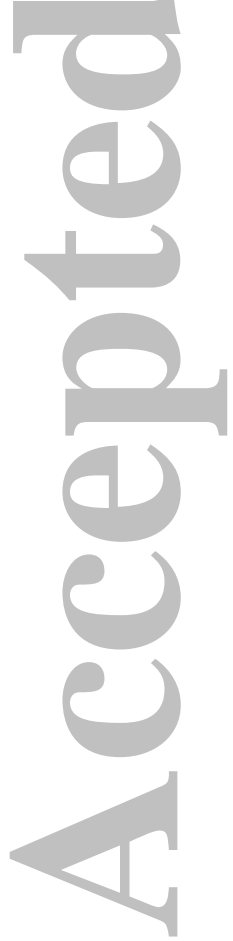


USG CARPAL TUNNEL RELEASE 16

\section{Tables}

Table 1. Boston Carpal Tunnel Questionnaire Mean Scores

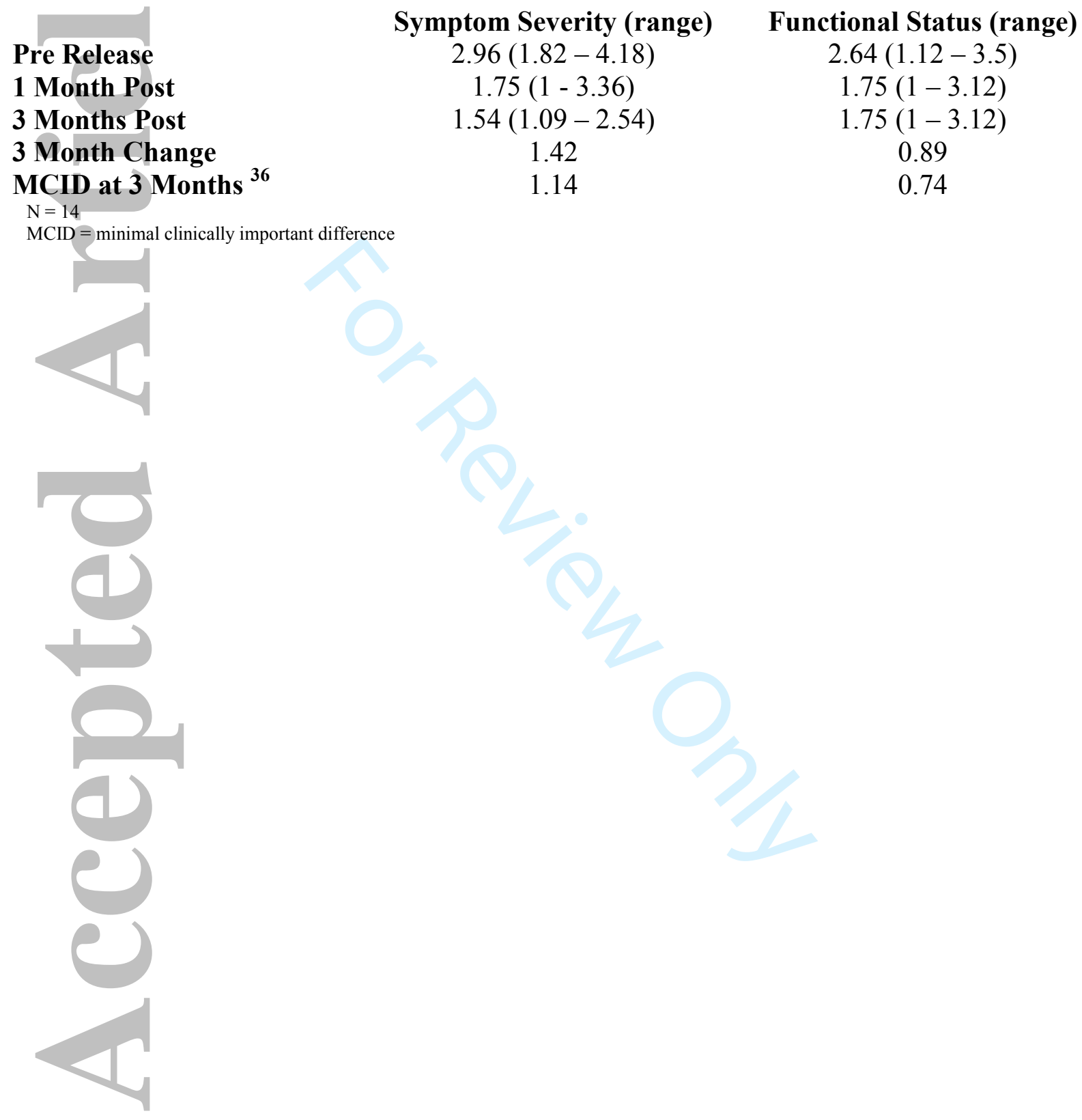


Table 2. QDASH Mean Scores

(1)

Pre-Release

1 Month Post

3 Months Post

3 Month Change

$\mathrm{N}=14$

MCID score 34 or change in score $>20$
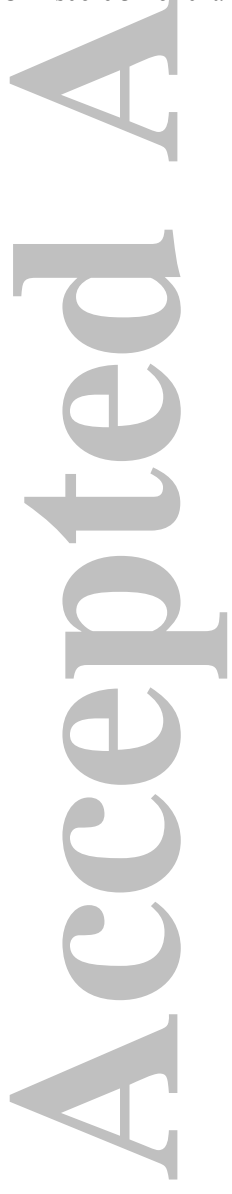

(range)

45

$(16-72)$

19

$(0-55)$

18

$(0-39)$

27

This article is protected by copyright. All rights reserved. 
USG CARPAL TUNNEL RELEASE 18

\section{Figure Legends}

\section{Figure 1. Pre-procedural Anatomic Markings and Skin Incision}

Picture of incision placement along the distal forearm and device direction of insertion (arrow).

Skin markings highlight the anatomic landmarks to assist during procedure. Blue line represents place for incision. Black dashed and solid red line marks location of the median nerve and ulnar artery respectively. a- superficial palmar arch; H- hook of hamate; P- pisiform, Mn- median nerve, U- ulnar nerve, S-scaphoid; T- Trapezium

\section{Figure 2. Placement of Device and Balloon Inflation}

A) External intra-operative photo of device placement. The device is initially inserted vertically until penetrating the antebrachial fascia, after which is advanced distally oblique-parallel to the forearm. B) In-plane view of the device within the carpal tunnel with proximal (PROX) to the left of the image. The device is placed $\sim 1 \mathrm{~cm}$ distal to the TCL (arrows) to ensure complete release. C) Out-of-plane view of the device (open arrow) within the transverse safe zone (dotted double arrow) at the level of the distal carpal tunnel (solid arrows). The protective balloons (curved arrows) are deflated as demonstrated in the insert. The TSZ in this patient is bordered by the ulnar artery, which encroaches in the tunnel. Therefore, the ULN side of arrow spans from the median nerve (dotted ellipse) to the ulnar artery $(a)$ and not the hook of the hamate $(H)$. D) Out-of plane view of the device with protective balloons (curved arrows) inflated as demonstrated in the insert. Note expansion of the TSZ (dotted double arrow) and the radial displacement of the median nerve (dotted ellipse). FT- flexor tendons; Solid ellipse- ulnar nerve; ThM- Thenar muscles; ULN- ulnar 


\section{Figure 3. Ultrasound-Guided Release of the Transverse Carpal Ligament}

A) External picture of the device with protective balloons inflated and the retrograde cutting knife (open arrow) deployed. B) Correlative US in-plane view of the device with distal (DIST) to the right of the knife (open arrow) cutting the TCL (solid arrows)

Figure 4. Ultrasound Confirmation of Complete Release of the Transverse Carpal

\section{Ligament}

Out of plane view of device (open arrow), with the protective balloons (curved arrows) deflated, showing the device superficial to the boundaries of the transected TCL (solid arrows).

a- ulnar artery; FCR- flexor carpi radialis - FT- flexor tendons; Rad- radial; S- scaphoid; Solid ellipse-ulnar nerve; ThM- Thenar muscles; ULN- ulnar

\section{Figure 5. Post-procedure wound closure}

Intraoperative photograph displaying closed incision with a single suture. 
USG CARPAL TUNNEL RELEASE 20

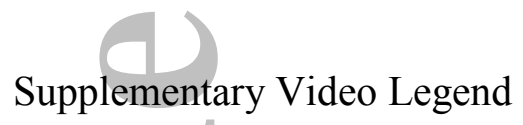

Supplementary Video 1: Balloon Inflation:

Out-of plane US image of inflation of the protective balloons (curved arrows) within the TSZ (dotted double arrow) with ulnar (ULN) to the right of the image. The TSZ in this patient is bordered by the ulnar artery, which encroaches in the tunnel. Therefore, the ULN side of arrow spans from the median nerve (dotted ellipse) to the ulnar artery $(a)$ and not the hook of the hamate $(H)$. Note expansion of the TSZ and the radial displacement of the median nerve (dotted ellipse).

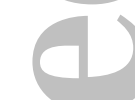

\section{Supplementary Video 2: Ultrasound-Guided Carpal Tunnel Release}

US in-plane view of the device with distal (DIST) to the left, of the microknife (open arrow) cutting the TCL (solid arrows) from a distal-to-proximal direction.

\section{Supplementary Video 3: Confirming Complete Ultrasound-Guided Carpal Tunnel Release}

Out of plane view of device (open arrow) with radial to the right of the image, with the

protective balloons (curved arrows) deflated, showing the device superficial to the boundaries of the transected TCL (solid arrows). 


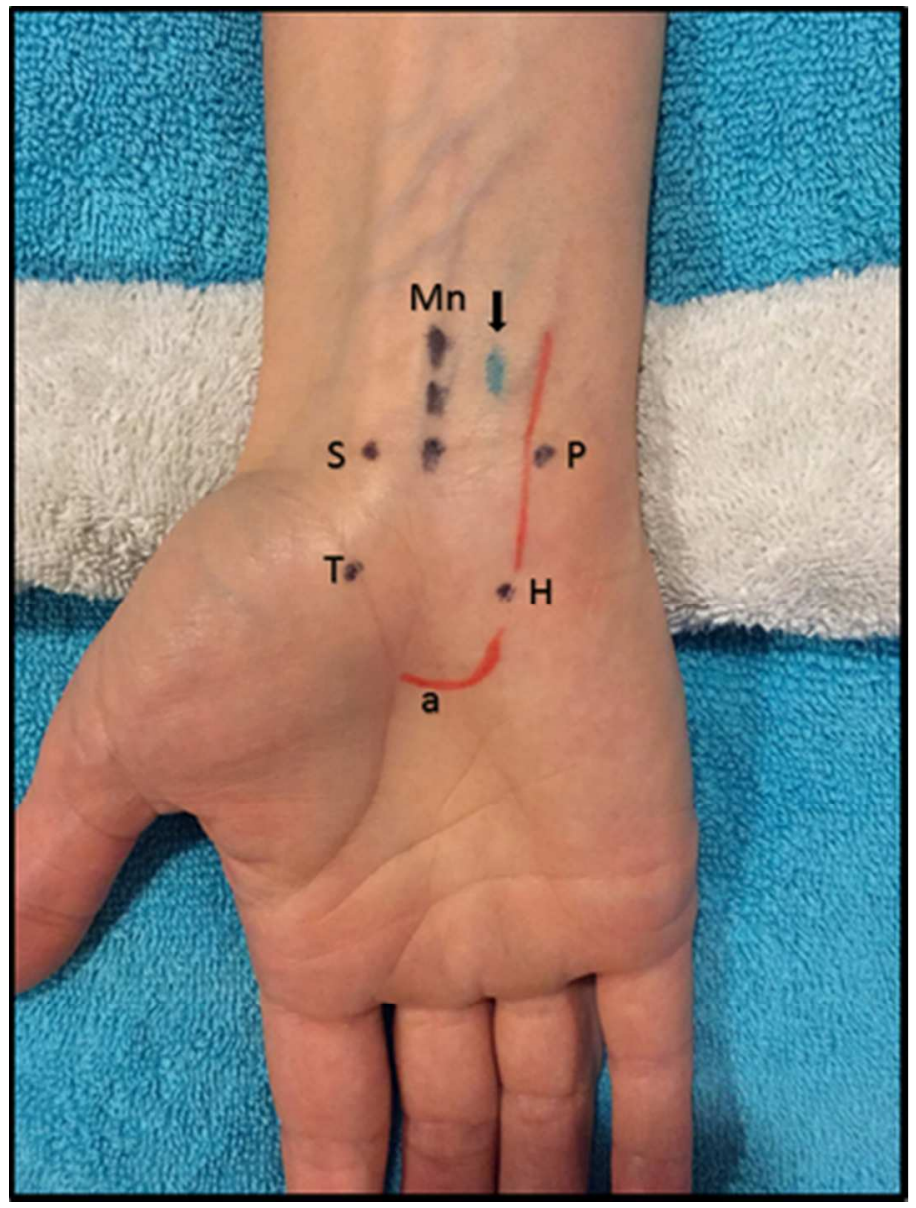

Figure 1. Pre-procedural Anatomic Markings and Skin Incision Picture of incision placement along the distal forearm and device direction of insertion (arrow). Skin markings highlight the anatomic landmarks to assist during procedure. Blue line represents place for incision. Black dashed and solid red line marks location of the median nerve and ulnar artery respectively. a- superficial palmar arch; $\mathrm{H}$ - hook of hamate; P- pisiform, $\mathrm{Mn}$ - median nerve, U- ulnar nerve, S-scaphoid; T- Trapezium

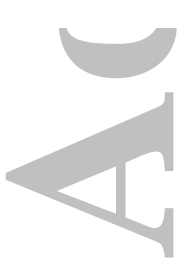

$29 \times 38 \mathrm{~mm}(300 \times 300 \mathrm{DPI})$ 


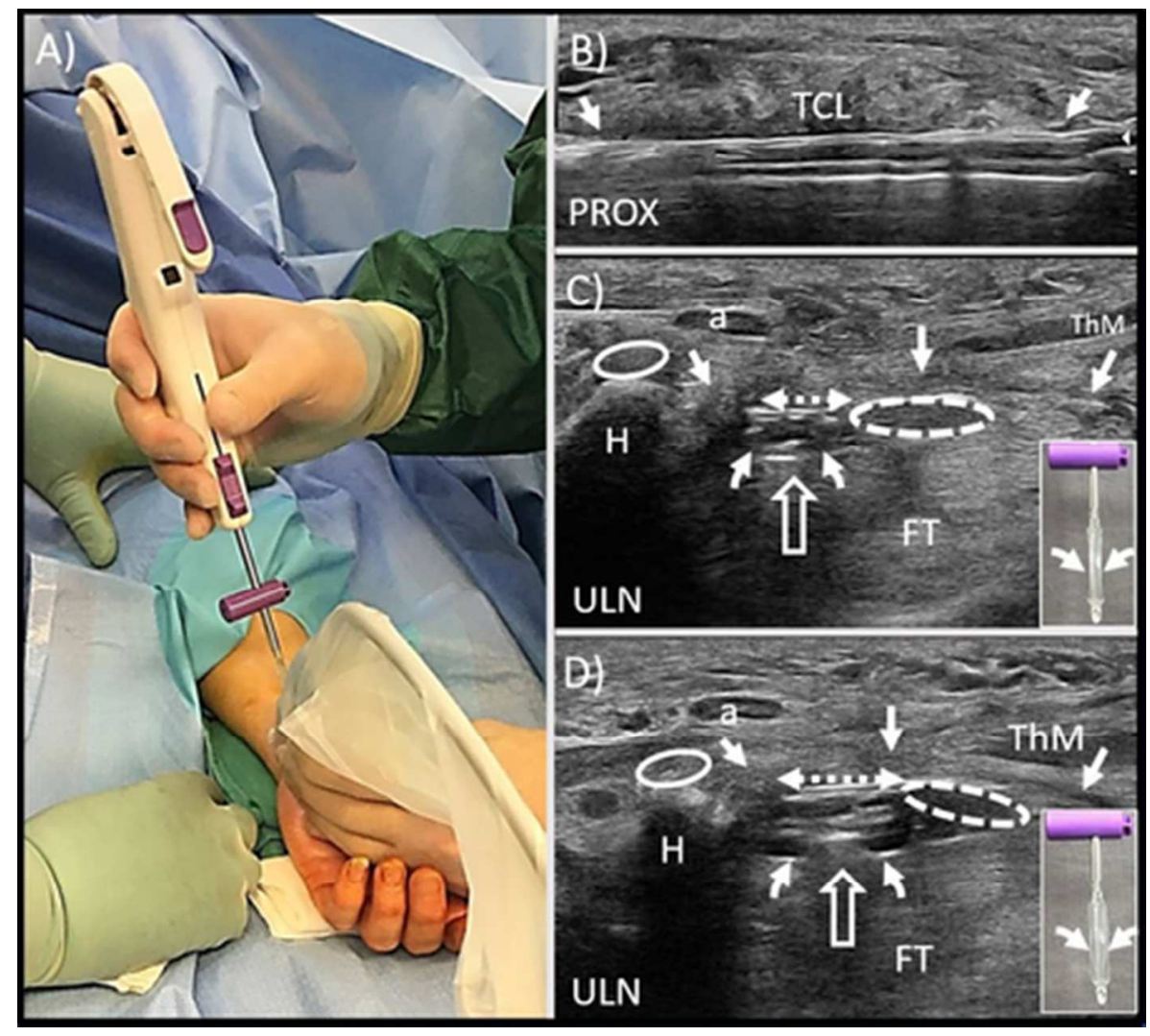

Figure 2. Placement of Device and Balloon Inflation

A) External intra3operative photo of device placement. The device is initially inserted vertically until penetrating the antebrachial fascia, after which is advanced distally oblique-parallel to the forearm. B) Inplane view of the device within the carpal tunnel with proximal (PROX) to the left of the image. The device is placed $\sim 1 \mathrm{~cm}$ distal to the TCL (arrows) to ensure complete release. C) Out-of-plane view of the device (open arrow) within the transverse safe zone (dotted double arrow) at the level of the distal carpal tunnel (solid arrows). The protective balloons (curved arrows) are deflated as demonstrated in the insert. The TSZ in this patient is bordered by the ulnar artery, which encroaches in the tunnel. Therefore, the ULN side of arrow spans from the median nerve (dotted ellipse) to the ulnar artery (a) and not the hook of the hamate $(H) . D)$

Out-of plane view of the device with protective balloons (curved arrows) inflated as demonstrated in the insert. Note expansion of the TSZ (dotted double arrow) and the radial displacement of the median nerve (dotted ellipse). FT- flexor tendons;

Solid ellipse - ulnar nerve; ThM- Thenar muscles; ULN- ulna

$38 \times 34 \mathrm{~mm}(300 \times 300 \mathrm{DPI})$ 


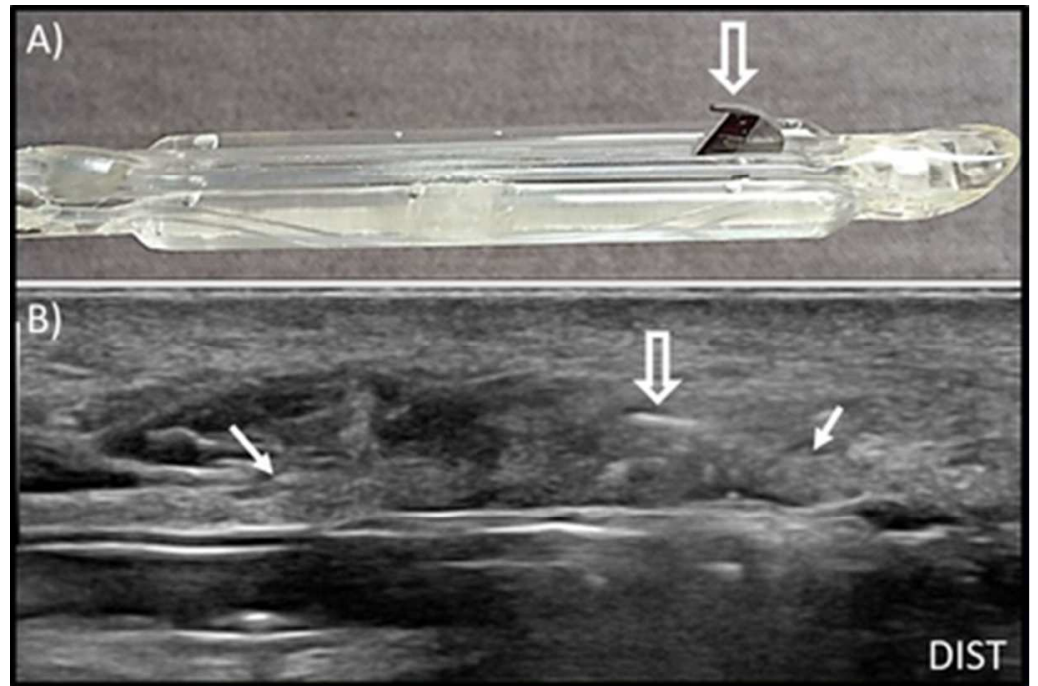

Figure 3. Ultrasound-Guided Release of the Transverse Carpal Ligament A) External picture of the device with protective balloons inflated and the retrograde cutting knife (open arrow) deployed. B) Correlative US in-plane view of the device with distal (DIST to the right of the knife (open arrow) cutting the TCL (solid arrows)

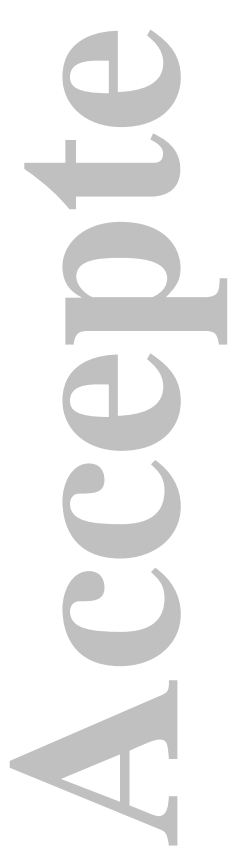

$33 \times 22 \mathrm{~mm}(300 \times 300 \mathrm{DPI})$ 


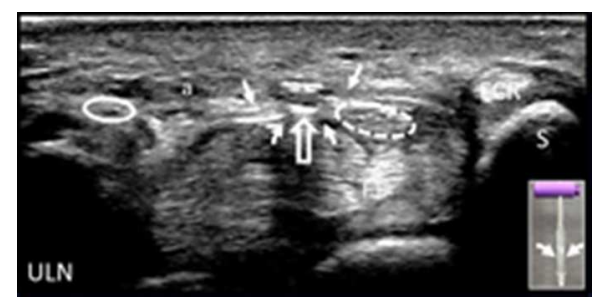

Figure 4. Ultrasound Confirmation of Complete Release of the Transverse Carpal Ligament Out of plane view of device (open arrow)

, with the protective balloons (curved arrows) deflated, showing the device superficial to the boundaries of the transected TCL (solid arrows).

a- ulnar artery; FCR- flexor carpi radialis - FT flexor tendons; Rad- radial; S- scaphoid; Solid ellipse - ulnar nerve; ThM- Thenar muscles; ULN- ulnar

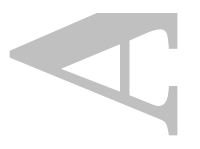

$18 \times 9 \mathrm{~mm}(300 \times 300 \mathrm{DPI})$

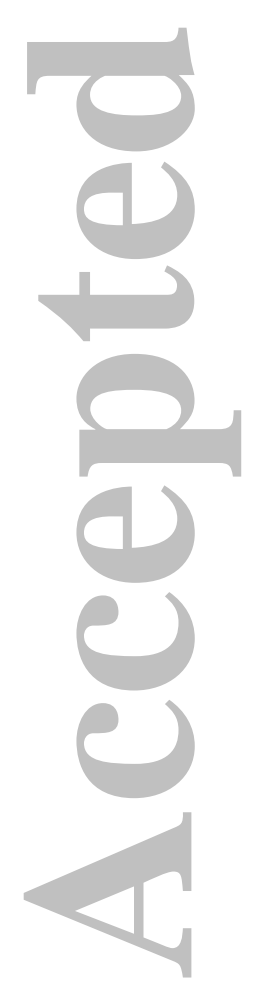




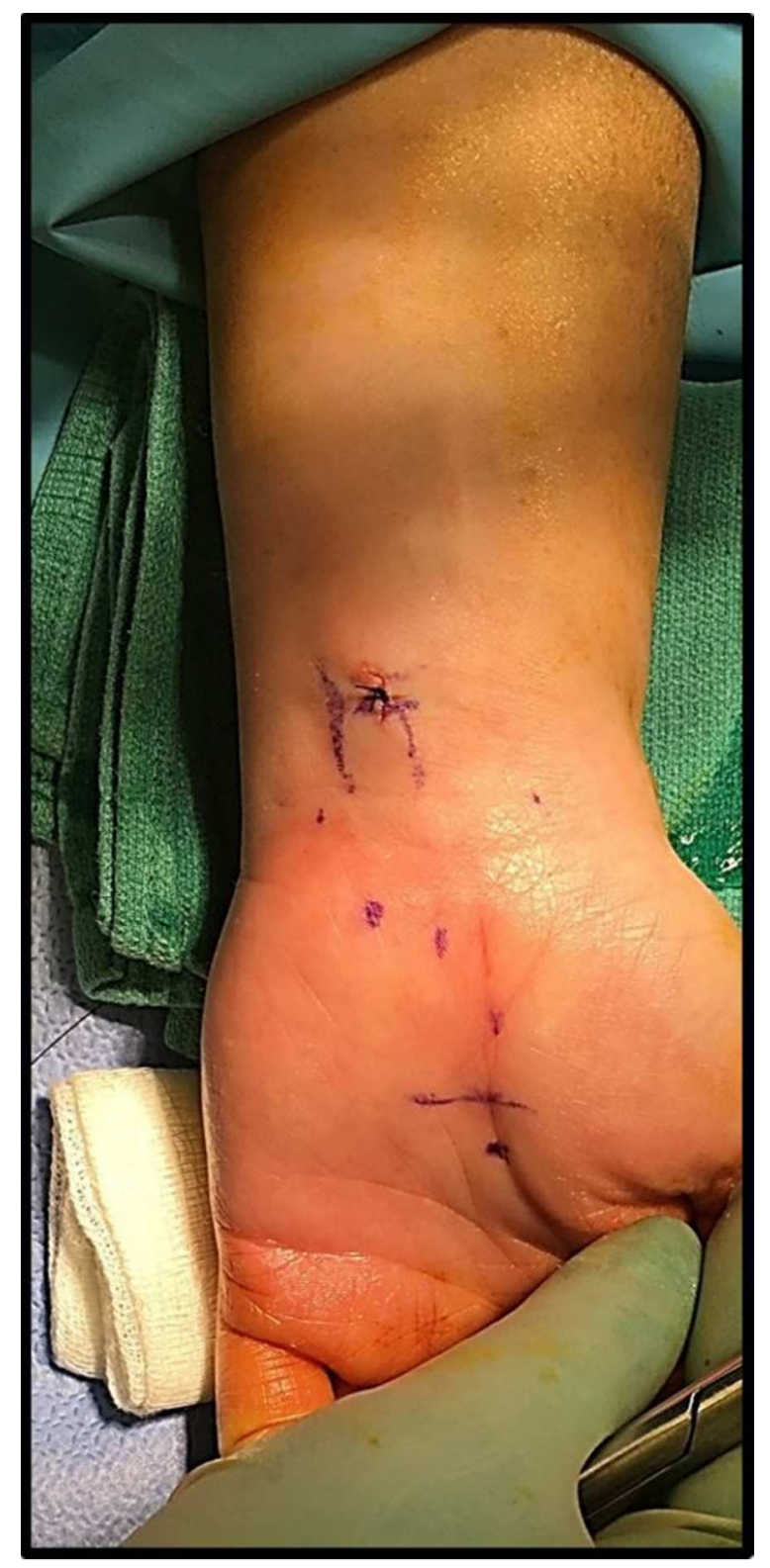

Figure 5. Intraoperative photograph displaying closed incision with a single suture. $41 \times 87 \mathrm{~mm}(300 \times 300 \mathrm{DPI})$ 